\title{
CEU? PIC
}

CENTRO UNIVERSITÁRIO DE BRASÍLIA - CEUB

PROGRAMA DE INICIAÇÃO CIENTÍFICA

EDUARDO FELIPE NÁCUL

ANÁLISE COMPARATIVA DAS MODALIDADES DE TRATAMENTO COM O MINOXIDIL TÓPICO E COMO O PRP PARA ALOPÉCIA ANDROGENÉTICA

BRASÍLIA

2021 


\title{
CEUP $\mid$ PIC
}

\section{EDUARDO FELIPE NÁCUL}

\section{ANÁLISE COMPARATIVA DAS MODALIDADES DE TRATAMENTO COM O MINOXIDIL TÓPICO E COMO O PRP PARA ALOPÉCIA ANDROGENÉTICA}

\author{
Relatório final de pesquisa de iniciação \\ científica apresentado à Assessoria de Pós- \\ Graduação e Pesquisa.
}

Orientação: Dr. Márcio Rabelo Mota. 


\section{DEDICATÓRIA}

A todos os profissionais da área da saúde, biólogo e enfermeira, envolvidos na elaboração do projeto. A todos que forneceram recursos humanos, recursos de locação, material e tempo para a realização da pesquisa. Aos amigos e familiares envolvidos. Aos pacientes, que por meio de coragem e fé, contribuíram para a realização deste projeto. 


\section{AGRADECIMENTO}

Agradeço ao CEUB pela oportunidade fornecida aos alunos de terem contato com programas de Iniciação Científica e outras atividades de extensão. A FAP-DF por terem investido no nosso projeto. Ao Dr. Márcio Rabelo Mota pelos recursos materiais fornecidos e pela ajuda em toda a parte prática e acadêmica. A enfermeira Mayara Silva por toda atenção dada em relação a agendamento dos pacientes, coleta de dados, execução dos procedimentos e pela disponibilidade e atenção. Ao colega Vitor Falqueto por ter idealizado parte do projeto comigo em um momento crítico, que era o envio dos projetos para a seletiva do PIC. A minha irmã Fernanda Nácul e a sua colega Luiza Giarola por terem nos auxiliado em diversos momentos durante o projeto. A Maria Luiza Santos e Anna Carolina Lengruber pela parceria e colaboração. Aos meus amigos pelo apoio e incentivo. 
"Nós nos transformamos naquilo que praticamos com frequência. A perfeição, portanto, não é um ato isolado. É um hábito."

Aristóteles, 300 a.C. 


\section{RESUMO}

A alopecia androgenética (calvície) é uma das dez maiores queixas nos consultórios dermatológicos e acometem, principalmente, os homens de 18 a 60 anos de idade. 0 impacto dessa condição clínica não é apenas estético, mas sim nos âmbitos psicológico, social, do trabalho, de estudo e etc. Dessa forma, a necessidade de tratamento é extremamente relevante e, portanto, existem diversas opções terapêuticas. 0 tratamento objetivo a redução ou controle da queda e miniaturização do cabelo. Dentre eles, o Plasma Enriquecido em Plaquetas (PRP) e o Minoxidil tópico a 5 \% são muito promissores. O PRP é utilizado para diversas outras condições clínicas e cirúrgicas, como no processo de cicatrização, cirurgias ortopédicas e procedimentos dermatológicos. Por sua vez, o Minoxidil é amplamente utilizado para controlar a queda de cabelo e tentar promover o seu crescimento. O objetivo desse estudo é, portanto, comparar a eficácia de ambos os tratamentos para a Alopécia Androgenética em indivíduos do sexo masculino com idade igual ou superior a 18 e até a idade igual ou inferior a 60 anos. Dessa forma, foi realizado um estudo randomizado e sem cegamento, no qual 13 pacientes foram submetidos a uma das duas formas de tratamento (Plasma Enriquecido em Plaquetas ou minoxidil tópico a 5\%). 4 receberam o minoxidil tópico a 5\% por 1 mês e ou outros 9 receberam o Plasma Enriquecido em Plaquetas a cada 21 dias, totalizando 3 aplicações. Ademais, foram analisados, por meio de anamneses estruturadas e por meio de questionários de autoavaliação, os aspectos psicológicos, sociais e biológicos de ambos os tratamentos.

Palavras-chave: alopécia androgenética; minoxidil; plasma enriquecido em plaqueta. 
SUMÁRIO

1 INTRODUÇÃO

2 FUNDAMENTAÇÃO TEÓRICA 9

3 MÉTODO 12

4 RESULTADOS E DISCUSSÃO

5 CONSIDERAÇÕES FINAIS (OU CONCLUSÕES) 17

$\begin{array}{ll}\text { REFERÊNCIAS } & 17\end{array}$

$\begin{array}{ll}\text { ANEXOS } & 20\end{array}$ 


\section{INTRODUÇÃO:}

A alopecia androgenética (AAG) é a forma mais comum de alopecia (calvície) em ambos os sexos. O último censo da Sociedade Brasileira de Dermatologia demonstra que está entre as dez queixas mais frequentes no consultório dermatológico em pacientes de 15 a 39 anos. Mais de $50 \%$ dos pacientes apresentam calvície acima dos 50 anos. (MULINARI-BRENNER, 2011).

Nesse contexto, diversas medidas farmacológicas são propostas para o tratamento da Alopécia Androgenética, dentre as quais se destacam: finasterida via oral e minoxidil tópico em concentações de 5\%. Ademais, associa-se a esses fármaco, mudanças no estilo de vida (controle de peso, diminuição da glicemia e melhoramento do perfil hormonal). Entretanto, são de uso crônico, não restauram totalmente o cabelo e apresentam efeitos colaterais (MULINARI-BRENNER, 2011; SENNET, 2019).

O plasma enriquecido em plaquetas, concentrado autólogo de plaquetas, o qual secreta fatores de crescimento para diversas células, se tornou comum para o tratamento de diversas condições de saúde. A sua popularidade ocorreu por ser um procedimento minimamente invasivo e com custo baixo. $O$ interesse no PRP surgiu em 2006 quando Mishra e Pavelko demonstraram a eficácia no tratamento para epicondilite. Dessa forma, se tornou um grande alvo para estudos no tratamento da alopecia androgenética, uma doença comum com grandes repercussões sociais. (Giordano et. Al, 2017).

Nesse ínterim, cientistas internacionais e nacionais, como do Departamento de Dermatologia da Northeast Ohio Medical University, Departamento de Dermatologia da Universidade de Aswan no Egito, Departamento de Dermatologia, Venereologia e Lepra da Faculdade Médica Smt. Kashibai Navale na Índia, Sociedade Americana de Cirurgia Dermatológica, Hospital Clementino Filho no Rio de Janeiro e outros diversos pesquisadores no mundo tem estudado um novo tratamento, mais inofensivo, altamente eficaz e próspero, o plasma enriquecido de plaquetas (PRP) para o tratamento da doença (TAIEB, 2016; STEVENS, 2018; ALVES, 2016; VENDRAMIN, 2009).

Uma metanálise, que analisou 177 pacientes de 6 estudos diferentes, encontrou resultados positivos no crescimento de cabelo dos pacientes submetidos ao PRP, em relação ao controle. $O$ grupo do PRP obteve um engrossamento na espessura do cabelo e na quantidade. (Giordano et. Al, 2017)

A maioria dos estudos encontrados analisaram a eficácia do PRP em relação a alopécia androgenética; entretanto, poucos comparam os tratamentos mais comumente utilizado na prática clínica com a alopécia e, os que os fazem, comparam com a Alopécia Areata (outro tipo de perda de cabelo). Para a realidade brasileira e mais especificamente a do Distrito Federal (Brasil), a alopecia androgenética é a mais relevante (MULINARI-BRENNER, 2011).

Em suma, o presente projeto de pesquisa tem o objetivo de realizar uma comparação entre o tratamento da AAG com o Minoxidil tópico $5 \%$ e o com PRP adicionado de ozônio, englobando os espectros biológicos, psicológicos e sociais e aplicados na realidade brasileira. Avaliar a eficácia do tratamento da AAG com Minoxidil por meio de uma anamnese, autoavaliação, inspeção, fotografia e dermatoscopia. Avaliar a eficácia do tratamento da AAG com Plasma Rico em Plaquetas por meio de uma anamnese, autoavaliação, inspeção, fotografia e dermatoscopia. Fazer uma autoavaliação do paciente em relação ao tratamento com Minoxidil a 5\% tópico, por meio de um questionário que avalie os impactos sociais da doença na vida do paciente, 
reações adversas com o tratamento (sinais flogísticos, nenhum sintoma ou outros sintomas), expectativas e grau de satisfação com o tratamento. Fazer uma autoavaliação do paciente em relação ao tratamento com PRP, por meio de um questionário que avalie os impactos sociais da doença na vida do paciente, reações adversas com o tratamento (sinais flogísticos, nenhum sintoma ou outros sintomas), expectativas e grau de satisfação com o tratamento. Comparar os dados da autoavaliação dos pacientes tratados com PRP e com o Minoxidil e entender as consequências psicológicas atribuídas a cada uma das opções e estabelecer diferenças, se existentes, entre elas. Analisar, sintetizar e cruzar os dados recolhido de ambos os protocolos de tratamento, para levantar dados sobre a eficácia dos tratamentos com PRP e com o Minoxidil tópico a 5\%. Associar os dados biológicos/técnicos do tratamento da AAG, obtidos pela avaliação clínica, com os psicossociais obtidos pelos questionários. Trazer visibilidade ao tema da AAG para a sociedade brasileira, promovendo avanços nos conhecimentos acerca das possibilidades terapêuticas relacionadas à doença para a área da dermatologia, tricologia, estética e psicologia.

\section{FUNDAMENTAÇÃO TEÓRICA:}

A Alopécia denomina as diferentes formas de perda de cabelo, os quais podem ter diversas causas. A depender do mecanismos fisiopatológico subjacente e da apresentação clínica, pode ser classificada em alopecia areata, eflúvio telógeno, eflúvio anageno, alopecia androgenética, tricotilomania e entre outras. A mais comum é a alopecia androgenética. Embora não seja comprometedora para a manutenção da vida do paciente, está relacionada a diminuição na qualidade de vida. (PHILLIPS et. Al, 2017)

Em relação ao aspecto sociocultural, cada cultura, etnia e grupos sociais atribuem ao cabelo simbolismo e significado histórico. Os budistas raspam seus cabelos como forma de anunciar o celibato; já as freiras e muçulmanas os cobrem como forma de se resguardar, porque o consideram um componente muito íntimo do corpo humano (procuram "manter a modéstia"). Por sua vez, a etnia negra, por meio do estilo de cabelo, a exemplo do black power e dread lock, o utilizaram como forma de se firmar na sociedade como indivíduos dotados de cultura, história e independentes, para que pudessem combater o racismo vigente na sociedade. Por fim, em todas as outras culturas no século XXI, independente da raça, vem se preocupando cada vez mais com o estilo de cabelo e estética no geral (GOMES, 2002).

Além disso, no âmbito do aspecto psicológico, os pacientes que sofrem com a perda de cabelo podem ter sua autoestima afetada, além de provocar alterações nos fatores emocionais, comprometendo a qualidade de vida. Logo, os tratamentos que afetam diretamente na qualidade da saúde capilar dos indivíduos, é crucial para a melhora da autoestima e da qualidade de vida (NANTES; 2018). Em um estudo realizado com mulheres com AAG, 88\% mulheres referem efeitos negativos em sua vida decorrentes da queda de cabelos, $75 \%$ apresentam baixa autoestima, e $50 \%$ problemas sociais (MULINARI- BRENNER et.al., 2011). Um dos fatores que podem estar associados com isso é que muitos homens e mulheres não conseguem aceitar as mudanças que vem com o envelhecimento, os quais pode-se citar a perda de cabelo, o aparecimento de rugas, o aparecimento de pelos brancos, e, por isso, procuraram formas de rejuvenescer, por meio de tratamentos médicos (CASTRO, 2016).

Por fim, em relação aos aspectos biológicos, fornece uma proteção ao couro cabeludo contra os raios UV, pelo fato de ser uma das regiões do corpo em que a pele 
mais fica exposta aos raios solares potencialmente danosos. Ademais, o cabelo se adapta às condições climáticas do meio, e também, participa de mecanismos fisiológicos responsável por fornecer informações, por meio de mecanoceptores, ao sistema nervoso central pelo tato protopático (SENNET, 2019). Em suma, condições que levam a queda de cabelo, denominada alopécia, embora não potencialmente fatal, podem influenciar as relações interpessoais e sociais dos indivíduos acometidos (VOGT et al., 2008).

O cabelo é formado pelos folículos pilosos, que consistem em um (mini)órgão cuja densidade capilar, distribuição futura e cor são características definidas geneticamente. De forma geral, é composto por um músculo eretor do pelo, por uma glândula sebácea e por um pelo. O pelo propriamente dito apresenta várias camadas: córtex, bainha externa, canal folicular, cutícula, medula, bulbo, zona queratinógena e pelo queratinizado. Seu crescimento depende de diversos fatores bioquímicos e é dividido em quatro fases: anágena (crescimento), catágena (regressão), quiescência (telógena) e desprendimento (exógena). Alguma alteração em uma dessas fases podem ocasionar a perda de cabelo (NANTES et al., 2018; SENNET, 2019)

A alopecia androgenética, também conhecida como perda de cabelo com padrão masculino ou calvície, é uma perda de cabelo mediada pelo hormônio dihidrotestosterona (DHT). Está também fortemente relacionada a fatores genéticos. Seu efeito clínico é a miniaturização dos pelos. A apresentação clínica varia do homem para a mulher. No sexo masculino, a perda de cabelo inicia na região frontal e evolui para a região temporal. No sexo feminino, diminuição de forma difusa na região centroparietal. (ADIL E GODWIN, 2017; NANTES et al., 2018; SENNET, 2019 ; BRENNER et al., 2011; STEVENS et al., 2018; )

A epidemiologia da Alopécia Androgenética aponta ser a forma mais frequente de calvície em ambos os sexos. Apresenta prevalência crescente conforme a idade. De forma geral, inicia entre 15 e 25 anos de idade nos homens, sendo que aparece antes dos 17 anos em $14 \%$ do total de homens com AAG, e 25 e 30 anos de idade nas mulheres. Outros dados indicam que $50 \%$ dos homens apresentarão AAG aos 50 anos de idade e $80 \%$ aos 80 anos e $30 \%$ das mulheres aos 70 anos de idade (WHITING, 2006; MULINARI-BRENNER et al., 2011; SENNET, 2019).

No Brasil, estima-se que a quantidade de pessoas com AAG é de 42 milhões de pessoas (MULINARI-BRENNER et al., 2011). Mais especificamente, de acordo com a Sociedade Brasileira de Dermatologia, a faixa etária de 15 a 39 anos de idade, em território nacional, está relacionada com a maior frequência nos consultórios dermatológicos. Por fim, percebe-se que a maior parte das pessoas acometidas são homens de 20 a 60 anos de idade e no DF, a porcentagem de homens nessa faixa etária é de aproximadamente $62,9 \%$.

A etiopatogenia está associada a fatores hormonais (androgênicos), genéticos e de estilo de vida/ambiental, a citar o tabagismo, hiperlipidemia, consumo excessivo de álcool. Os fatores genéticos envolvidos ainda são pouco conhecidos, porém há evidências suficientes que confirmam o seu envolvimento. $O$ padrão de herança é poligênico (ex., EDA2R, ERb e o da síndrome dos ovários policísticos). A fisiopatologia, portanto, consiste numa alteração do ciclo do folículo piloso. Dessa forma, ocorre o término prematuro da fase anágena (crescimento) pela redução da expressão de fatores estimulantes e aumento de citocinas que promovem apoptose, que promove a fase catágena (regressão). Todos esses fatores resultam em miniaturização folicular 
progressiva, com transformação de fios terminais em velos (pelos mais curtos, finos e menos pigmentados) (NANTES et al., 2018; SENNET, 2019 ; BRENNER et al., 2011; STEVENS et al., 2018).

A AAG no homem está relacionada principalemte a testosterona. A DHT é um metabólito responspavel pela minitaturização dos folículos e desenvolvimento da doença. Embora seja um processo DHT-dependente nos homens, a maioria apresenta níveis normais de testosterona. Ocorre em indivíduos geneticamente predispostos. (BRENNER et. al, 2011).

O diagnóstico é clínico e não há um método padrão-ouro. Uma anamnese completa deve ser realizada, na qual avalia a variações de peso, uso de anabolizantes, hábitos alimentares, uso de produtos químicos, medicações, história familiar e comorbidades. O principal diagnóstico diferencial é o eflúvio telógeno. Métodos complementares podem ser realizados pela dermatoscopia, tricograma e biópsia. (BRENNER et. al, 2011)

O objetivo do tratamento é prevenir a evolução da queda, estabilizar e reverter o processo de miniaturização e aumentar a densidade capilar. Deve-se, junto ao tratamento, padronizar a coleta de fotos para avaliação prognóstica e decisão terapêutica. O tratamento é farmacológico e não farmacológico. Dieta balanceada, tratamento de outras doenças do couro cabeludo, diminuição do peso, aumento da sensibilidade à glicose e controle glicêmico, aumento do SHBG e redução da restosterona live devem ser adotadas como medidas não farmacológicas. Nenhuma das drogas restauram toda a perda capilar. Há diversas opções disponíveis, como a flutamida, a dunasterida, espirolactona, finasterida, minoxidil e suplementação de vitaminas e ferro. (SENNET, 2019; BRENNER et al., 2011)

O minoxidil é um potente vasodilatador, antes utilizado para o controle da Hipertensão Arterial Sistêmica, porém hoje utilizado como um dos principais recursos terapêuticos da Alopécia Androgenética. A sua ação ainda não está totalmente esclarecida, porém estudos indicam que promove o aumento do nível de atividade do fator de crescimento endotelial vascular (FCEV) e vasodilatação local. Dessa forma, diminui a fase telogênica do ciclo capilar e os mantém anágenos, o que leva a um aumento do diâmetro do cabelo, especialmente daqueles que foram miniaturizados. 0 pico de ação é notado por volta de 16 semanas de uso, e após seis meses de descontinuação o quadro volta a como era antes. A utilização da solução de minoxidil tópica aprovada é referente à administração de uma dosagem de $1 \mathrm{~mL}$ sobre o couro cabeludo seco, duas vezes por dia, tendo sido verificado que uma solução de $5 \%$ de minoxidil é mais eficaz do que $2 \%$ ou $3 \%$. Ademais são quase raros os efeitos colaterais, os quais podem ser dermatite, hipertricose, prurido, irritação local e hipertricose (SENNET, 2019; BRENNER et al., 2011; NANTES et al., 2018).

O PRP (plasma enriquecido de plaquetas) é um produto autólogo (derivado do paciente), obtido laboratorialmente, que fornece fatores de crescimento por meio um processo de centrifugação do sangue. É um procedimento que vem sendo cada vez pesquisado e utilizado por pesquisadores e clínicos em todo o mundo e apresentando excelentes resultados no tratamento da AAG. A técnica apresenta diversas áreas de utilização, dentre os quais a odontologia, pé diabético, cirurgia plástica, pois apresenta propriedades adesivas, hemostáticas e cicatrizantes. É um hemocomponente composto de plasma concentrado em plaquetas. Quando adicionado de trombina e cálcio, resulta na ativação das plaquetas, que é rico em fatores de crescimento como o PDGF, TGF- 
beta, EGF, VEGF e IGF-1 STEVENS et.al., 2018; VASCONCELOS et.al., 2015; NANTES et.al., 2018).

No tratamento do AAG, os fatores de crescimento estimulam as células da região bulge a manter a fase de crescimento (anágena) mais longa, reduzindo também o processo inflamatório e apoptose. Isso ocorre pois, o PRP, ao ser injetado no couro cabeludo, ativa fibroblastos locais, promove a síntese de colágeno, estimula a matriz extracelular e aumenta, também, a expressão de fatores de crescimento endógeno. Dessa forma, estimulam as células da região do bulge a dar origem mais uma vez a um folículo piloso. Outrossim, podem promover a angiogênese, por meio dos fatores angiogênicos liberados, que aumenta o aporte de oxigênio e nutrientes para a unidade folicular, diferenciação celular e quimiotaxia. Os benefícios do PRP para o tratamento de AAG é que é seguro, não apresenta risco de incompatibilidade (é autólogo), apresenta mínimos efeitos colaterais, como dor no momento da aplicação e eritema local (STEVENS et al., 2018; VASCONCELOS et al., 2015; NANTES et al., 2018).

\section{MÉTODO:}

\subsection{Tipificação:}

O estudo é do tipo experimental de ensaio clínico randomizado sem cegamento. Pacientes foram submetidos a duas modalidades distintas de tratamento para a Alopécia Androgenética e foram observados a resposta ao tratamento.

\subsection{Caracterização do local da pesquisa:}

Foi realizado na Clínica CER da Asa Sul, que se localiza no Centro Médico Julio Adnet, e na Clínica CER da unidade de Águas Claras. A clínica em questão realiza diversos procedimentos envolvendo ozonioterapia e procedimentos de outras naturezas, como acupuntura e PRP.

\subsection{Objeto de estudo:}

Foram estudados, no total, 13 pacientes homens de 18 a 60 anos com alopécia androgenética. 4 pacientes foram submetidos ao minoxidil tópico a $5 \%$ e outros 9 foram submetidos ao PRP (plasma enriquecido em plaquetas).

\subsection{Delimitação do universo da amostra:}

A amostra é de homens com idade de 18 a 60 anos. Foram excluídos os voluntários que: (1) Apresentavam infecções que possam afetar sua saúde capilar; (2) Apresentavam distúrbios psiquiátricos que prejudiquem o protocolo - como tricotilomania; (3) Que não foram cooperantes ou que apresentaram qualquer inflamação, eritema (exceto dermatite seborreica leve) ou cicatrizes no couro cabeludo; (4) Que utilizaram anticoagulantes, anti-hipertensivos, anticonvulsivantes, aspirina ou outros anti-inflamatórios não esteróides; (5) Que tinham história prévia de malignidades, distúrbios plaquetários ou hemorrágicos, aplasia da medula óssea, diabetes, HIV, infecção por hepatite B ou C ou imunocomprometidos; tinham propensão a quelóides.

\subsection{Instrumentos de coleta ou de geração de dados:}


Os dados foram coletados por meio de uma anamnese dirigida (anexo 1) e fotografias das regiões parietal, temporal, occipital e frontal da cabeça. Ademais, foi utilizado a tabela Hamilton-Norwood para comparar a evolução do paciente conforme os diferentes períodos de tratamento (Anexo 2). Os dados biopsicossociais fora coletados por meio de um questionário de autoavaliação (Anexo 3). Os dados coletados foram analisados e tabulados em uma planilha do excel para análise estatatística.

\subsection{Procedimentos metodológicos:}

Na primeira consulta, os participantes do projeto reberam informações sobre o projeto (local, datas, pessoas envolvidas, possíveis efeitos adversos, objetivos e resultados esperados). Após essa explicação, todos os pacientes receberam o TERMO DE CONSENTIMENTO LIVRE E ESCLARECIDO.

Após essa etapa, os pacientes que assinaram o TCLE, foram submetidos a uma anamnese criteriosa, feita pelo aluno bolsista. Cada consulta (desde a anamnese até o procedimento) demorou uma média de 60 minutos.

Após a anamnese (anexo 1 e 3), os participantes receberam o questionário de autoavaliação (anexo 2). Depois dessa etapa, foram submetidos ao exame físico, que consiste em: inspeção, dermatoscopia e fotografia.

1. A inspeção é um procedimento em que se avalia as regiões acometidas, a extensão, a presença de alguma outra alteração por meio do olhar criterioso. Os pacientes serão então classificados em um grau diferente de alopécia, de acordo com a extensão das áreas sem cabelo, como proposto por Hamilton e modificadas por Norwood em 1975, imagem no Anexo 2 (MULINARI-BRENNER, 2011). Essa classificação e foi importante para avaliar a evolução do tratamento.

2. A dermatoscopia é um exame rápido, não invasivo e capaz de fornecer dados importantes para o diagnóstico. Por meio deste, será avaliado o diâmetro do cabelo, densidade capilar e se houve alguma reação adversa local (ex., descamação ou eritema) em cada paciente antes de cada procedimento e em cada retorno.

3. A fotografia será feita por meio de uma câmera DSLR e usaremos para fazer um comparativo macroscópico e analisar a distribuição do cabelo de forma mais eficaz e clara, conforme a classificação disponível no anexo 2. Serão fotografados a região frontal, temporal, lateral e occipital de cada paciente. Todos os pacientes serão fotografados em todas as consultas logo antes do tratamento.

Feito o exame físico, os pacientes foram divididos aleatoriamente em 2 grupos, o que recebeu o Minoxidil Tópico a 5\% (protocolo 1) e o que recebeu o Plasma Enriquecido em Plaqueta (protocolo 2).

1. Protocolo 1: $O$ grupo 1 recebeu um tubo de minoxidil tópico a 5\% em gel, o qual deveria ser aplicado sobre o couro cabeludo, nas regiões acometidas pela AAG, após lavá-lo com água e sabão e secá-lo. O paciente deveria aplicar de 12 em 12 horas, diariamente, em sua residência ou outro local de referência, pelo próprio paciente ou ajudante, seguindo as orientações já citadas. O grupo 1 recebeu apenas 1 tubo e aplicou por 21 dias. Foi utilizado o minoxidil tópico a 5\% da marca Kirkland com tubos de $60 \mathrm{~mL}$. 
2. Protocolo 2: $O$ grupo 2 recebeu o gel de PRP, de 3 em 3 semanas (a cada 21 dias), totalizando 3 aplicações. No protocolo do Plasma Rico em Plaquetas foi realizado 3 aplicações a cada 21 dias. Em todas as sessões os voluntários passaram pelo processo de coleta de sangue, pela fossa cubital, para o processamento do PRP e infiltrações nas áreas acometidas logo em seguida. $O$ procedimento tinha duração média de 40 minutos por paciente. As infiltrações foram realizadas pela enfermeira, com material estéril e agulhas $30 \mathrm{G} 1 / 2$. Foram aplicados $0,2 \mathrm{ml}$ de PRP intradérmico em cada ponto da região acometida, com espaços em torno de $2 \mathrm{~cm}$ entre esses pontos. O volume de PRP processado foi individualizado de acordo com o tamanho da região a ser tratada em cada paciente. Foram utilizados os seguintes materiais para o protocolo PRP: Centrifugadora Kasvi (Indicada nas técnicas de Fibrina Rica em Plaquetas (PRP) e Plasma Rico em Fibrina (PRF) Modelo K14-0815a (aprovada pela Anvisa); aparelho de ozônio; seringas plásticas; tubos plásticos de citrato.

Todas as consultas foram realizadas da mesma forma, exceto no último retorno em que apenas os dados foram coletados (anamnese e exame físico), mas o procedimento não foi realizado.

\section{RESULTADOS E DISCUSSÃO:}

\begin{tabular}{|c|c|c|c|c|c|c|c|c|c|c|}
\hline \multicolumn{11}{|c|}{ DADOS COLETADOS DO PLASMA ENRIQUECIDO DE PLAQUETAS } \\
\hline \multirow{18}{*}{ 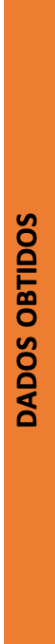 } & \multicolumn{10}{|c|}{ PACIENTES VOLUNTÁRIOS $(n=9)$} \\
\hline & \multirow[b]{2}{*}{ Expectativa do Tratamento } & \multirow{2}{*}{$\begin{array}{c}\text { Paciente } 1 \\
3\end{array}$} & \multirow{2}{*}{$\begin{array}{c}\text { Paciente } 2 \\
4\end{array}$} & \multirow{2}{*}{$\begin{array}{c}\text { Paciente } 3 \\
4\end{array}$} & \multirow{2}{*}{$\begin{array}{c}\text { Paciente } 4 \\
1\end{array}$} & \multirow{2}{*}{\begin{tabular}{|c|} 
Paciente 5 \\
4
\end{tabular}} & Paciente 6 & Paciente 7 & Paciente 8 & Paciente 9 \\
\hline & & & & & & & 4 & 3 & 4 & 2 \\
\hline & Psicológico e AAG & às vezes & Não & às vezes & Não & Sim & $\operatorname{Sim}$ & às vezes & Não & Sim \\
\hline & Aparência e AAG & Sim & Um pouco & às vezes & Sim & Sim & Sim & Sim & Sim & Sim \\
\hline & Trabalho ou facul e AAG & Não & Não & Não & Não & Não & Não & às vezes & Sim & às vezes \\
\hline & Socialização e AAG & Não & Não & Não & Não & Não & Não & Não & Não & Não \\
\hline & Vida Sexual e AAG & Não & Não & Não & Talvez & Não & Não & Não & Não & Não \\
\hline & Satisfação com o tto & 3 & 4 & 4 & 1 & 4 & 0 & 0 & 3 & 2 \\
\hline & Dificuldade no tratamento & Não & (Não/Não) & Não & Não & Não & Não & Não & Não & Não \\
\hline & Efeitos colaterais no tto & Não & Não & Dor local & Não & Vermelhidão & Dor local & Não & Não & Não \\
\hline & Alteração no cotidiano e Tto & Não & Não & Não & Não & $\operatorname{Sim}$ & Não & Não & Não & Não \\
\hline & Crescimento com a 19 aplicação & Mudou & $\mathrm{N}$ mudou & $\mathrm{N}$ mudou & Mudou & $\mathrm{N}$ mudou & Mudou & $\mathrm{N}$ mudou & $\mathrm{N}$ mudou & $\mathrm{N}$ mudou \\
\hline & Crescimento com a $2^{2}$ aplicação & Mudou & Mudou & Mudou & $\mathrm{N}$ mudou & Mudou & $\mathrm{N}$ mudou & $\mathrm{N}$ mudou & $\mathrm{N}$ mudou & Mudou \\
\hline & Crescimento com a 3a aplicação & Mudou & Mudou & Mudou & $\mathrm{N}$ mudou & Mudou & $\mathrm{N}$ mudou & $\mathrm{N}$ mudou & $\mathrm{N}$ mudou & Mudou \\
\hline & 0. Classificação de Hamilton e Norwood & III & III & $\|$ & III & IV & III & III & $\mathrm{VI}$ & v \\
\hline & 1. Classificação de Hamilton e Norwood & III & III & ॥ & III & IV & III & III & $\mathrm{VI}$ & $\mathrm{v}$ \\
\hline & 2. Classificação de Hamilton e Norwood & III & III & $\|$ & III & IV & III & III & $\mathrm{VI}$ & $\mathrm{V}$ \\
\hline
\end{tabular}

\section{Resultados dos voluntários do PRP (Plasma Enriquecido em Plaquetas):}

\section{a. Em relação aos aspectos biopsicossociais do PRP:}

Os dados para a comparação do tratamento da Alopécia Androgenética pelo minoxidil tópico a 5\% e para a alopecia androgenética foram tabulados para facilitar a análise comparativa.

Em relação à expectativa do tratamento, foi indagado, no primeiro dia do procedimento, qual a expectativa de 0 (nenhuma) a 5 (máxima) em relação ao Plasma Enriquecido em Plaquetas (PRP). 11\% deram a nota 1, 11\% deram a nota 2, 22\% deram 
a nota 3 e 55\% deram a nota 4 . Dessa forma, a maior parte dos pacientes estavam com expectativas elevadas em relação ao tratamento pelo PRP.

Foi indagado aos 9 pacientes do PRP se houve alguma alteração psicológica ocasionada pela calvície, principalmente de humor e autoestima. Aqueles pacientes que responderam "às vezes" foram colocados na categoria dos pacientes que afirmaram alterações psicológicas ("sim"). $66 \%$ dos pacientes responderam que desenvolveram ou que às vezes possuem alguma alteração psicológica decorrente da calvície. Por sua vez, $33 \%$ dos pacientes negaram que a calvície altera sua autoestima e seu humor.

A aparência foi outro ponto questionado acerca das alterações biopsicossociais dos voluntários do PRP. $100 \%$ respondeu que a calvície afeta de alguma forma o modo como se enxergam no mundo ou como imaginam que as pessoas a enxerga.

$33 \%$ dos voluntários desse grupo acreditam que a calvívie afetou, de algum modo, de forma negativa o seu ambiente de trabalho. Por outro lado, $66 \%$ acreditam que a calvívie não alterou o seu ambiente de trabalho, nem de forma negativa e nem de forma positiva.

Nenhum paciente (100\%) percebeu alteração em seu ambiente social (igreja, amigos, desconhecidos e etc.) decorrentes da alopecia androgenética.

Apenas 1 (11\%) paciente, dos 9 indagados, acredita que a calvície prejudicou de alguma forma a sua vida amorosa e/ou sexual.

\section{b. Em relação ao tratamento da AAG pelo PRP:}

Os pacientes submetidos ao tratamento com o Plasma Enriquecido em Plaquetas foram indagados após o retorno de 21 dias da última (terceira) aplicação em relação à satisfação com os resultados obtidos com o tratamento. Em uma escala de 0 (insatisfeito) a 5 (muito satisfeito). 33\% deu a "nota 4", 22\% deu a "nota 3", 22\% deu a "nota 0", $11 \%$ deu a "nota 2 " e $11 \%$ de a "nota 1 ".

Dos 9 voluntários, nenhum (100\%) tiveram alguma dificuldade em relação ao tratamento do Plasma Enriquecido em Plaquetas.

$66 \%$ dos pacientes não tiveram nenhum efeito colateral decorrente do tratamento. Entretanto, 33\% afirmaram algum efeito colateral, sendo que os sintomas relatados foram vermelhidão (1) e dor no local da aplicação (2).

Apenas 1 paciente (11\%) afirmou que o tratamento pelo PRP alterou de alguma forma o seu cotidianto. Os outros $8(88 \%)$ negaram qualquer alteração no cotidiano.

Em relação aos resultados obtidos com o tratamento e coletados por meio do dermatoscopio, foi analisado se houve mudança de uma aplicação para a subsequente. Os resultados tidos como positivo foram aqueles que houve uma diminuição na queda do cabelo ou crescimento de novos pelos nas regiões de aplicação. Após a primeira aplicação, 33\% obtiveram resultado positivo e $66 \%$ não obtiveram qualquer resultado. Após a segunda aplicação, 55\% dos participantes obtiveram alguma alteração e $44 \%$ não obtiveram. Após a 3ạ, e última aplicação, 55\% dos participantes notaram alguma alteração e $44 \%$ não.

A classificação de Hamilton e Norwood (H\&N) auxiliou para analisar mudanças macroscópicas, obtidas por uma simples inspeção, para avaliar resultados no tratamento. A partir dos dados coletados, conclui-se que nenhum voluntário obteve alteração na classificação de H\&N.

\section{Resultados dos voluntários do Minoxidil tópico à 5\%:}




\begin{tabular}{|l|c|c|c|c|c|}
\hline \multicolumn{5}{|c|}{ DADOS COLETADOS DO MINOXIDIL TÓPICO A 5\% } \\
\hline \multicolumn{1}{|c|}{ PACIENTES VOLUNTÁRIOS (n = 4) } \\
\hline
\end{tabular}

\section{a. Em relação aos aspectos biopsicossociais do Minoxidil tópico à 5\%:}

A avaliação da expectativa em relação ao tratamento pelo minoxidil tópico a $5 \%$ foi realizada na primeira consulta. Foi questionado, qual o resultado esperado, após 21 dias de aplicação, numa escala de 0 (nenhuma alteração) a 0 (muita alteração). 50\% dos voluntários deram a "nota 4" e 50\% deram a "nota 3".

$50 \%$ dos voluntários do minoxidil tópico à 55 acreditam que a calvície promoveu alguma alteração psicológica negativa (ex., baixa autoestima, depressão, ansiedade). Os outros $50 \%$ não acreditam que a calvície tenha influenciado negativamente seus psicológicos.

$50 \%$ dos voluntários acreditam que a AAG afetou negativamente a sua aparência. Por outro lado, os outros $50 \%$ não notaram qualquer alteração de aparência advinda da calvície.

$100 \%$ dos voluntários acreditam que a calvície não tenha influenciado no ambiente de faculdade ou de trabalho.

Em relação à vida social, foi questionado se a calvície alterou a forma de socialização, inserção em grupos sociais, criação de novas amizades ou interação com a sociedade. $25 \%$ dos participantes afirmaram que sim e $75 \%$ negaram qualquer influência da calvície nesse aspecto.

$75 \%$ participantes não notaram qualquer alteração na vida sexual ou amorosa decorrente da calvície. Entretanto, 25\% acredita que a alopecia androgenética alterou negativamente esse aspecto.

\section{b. Em relação aos aspectos biopsicossociais do Minoxidil tópico à $\mathbf{5 \%}$ :}

Os pacientes submetidos ao tratamento com o Minoxidil tópico a 5\% foram indagados após o retorno, de 21 dias utilizando o produto diariamente, em relação à satisfação com os resultados obtidos com o tratamento. Em uma escala de 0 (insatisfeito) a 5 (muito satisfeito). $75 \%$ dos voluntários deram a "nota 3" e 25\% dos voluntários deram a "nota 4."

$75 \%$ dos pacientes não relatar qualquer dificuldade em relação ao tratamento com o minoxidil tópico. Por outro lado, $25 \%$ relatou ter tido alguma dificuldade.

$50 \%$ dos voluntários relataram ter tido algum efeito colateral devido ao uso do tratamento e $50 \%$ relatou não ter tido qualquer tipo de efeito colateral. Dentre os efeitos colaterais relatados, esta a coceira e o eritema. 
Nenhum paciente (100\%) relatou qualquer alteração no cotidiano devido ao tratamento.

75\% dos pacientes notaram alguma alteração após 21 dias de uso. Entretanto, $25 \%$ não notou qualquer alteração.

\section{Considerações Finais:}

O estudo tinha como objetivo analisar comparativamente a eficácia e os aspectos biopsicossociais do tratamento da Alopécia Androgenética (calvície) por meio do Plasma Enriquecido em Plaquetas e do Minoxidil tópico a 5\%.

Em relação a eficácia do tratamento, o grupo do minoxidil ficou mais satisfeito em relação aos resultados esperados, do que o grupo do Plasma Enriquecido em Plaquetas. Em relação a adesão ao tratamento, observa-se que o grupo do minoxidil tópico obteve mais dificuldade em relação ao grupo do PRP. A explicação disso, em parte, pode ser devido a aplicação única do PRP a cada 21 dias e o minoxidil ter que ser aplicado duas vezes diariamente.

Os resultados obtidos após os primeiros 21 dias de apicação foi mais evidente no grupo do minoxidil tópico a $5 \%$ do que no do grupo do PRP.

Além disso, foi observado que a calvície afeta a vida social, a vida amorosa, a autoestima, a socialização dos portadores de alopecia androgenética.

Dentre as limitações do estudo, está a falta de cegamento e a população muito pequena. As discussões ainda não foram esgotadas, porém esse trabalho pode servir de inspiração e dar um norte para muitos estudos sobre o tratamento da alopecia androgenética.

\section{REFERÊNCIA}

VASCONCELOS, Rossana Cantanhede Farias de et al. A aplicação do plasma rico em plaquetas no tratamento da alopecia androgenética. Surgical and Cosmetic Dermatology , [s. I.], v. 7, n. 2, p. 130-137, 2015.

GIRIJALA, Raghavendra L et al. Platelet-rich plasma for androgenic alopecia treatment: a comprehensive review. Dermatology Online Journal, [s. I.], v. 24, n. 7, p. 1-13, 2018.

STEVENS, J. et al. Platelet-rich plasma for androgenetic alopecia: A review of the literature and proposed treatment protocol. International Journal of Women's Dermatology, [s. I.], p. 46-51, 2018.

SINGH, Satyendra Kumar et al. Comparison of efficacy of platelet-rich plasma therapy with or without topical $\mathbf{5 \%}$ minoxidil in male-type baldness: A randomized, doubleblind placebo control trial. Indian J Dermatol Venereol Leprol , [s. I.], v. 86, n. 2, p. 150157, 2020.

MULINARI-BRENNER, Fabiane et al. Entendendo a alopecia androgenética. Surgical and Cosmetic Dermatology , [s. I.], v. 3, n. 4, p. 329-337, 2011. 
CASTRO, Amanda et al. Representações sociais do envelhecimento e do rejuvenescimento para mulheres que adotam práticas de rejuvenescimento. Psico, [s. I.], v. 47, n. 4, p. 319-330, 2016.

NANTES, Mariana Correa et al. Ação do minoxidil e da finasterida através da intradermoterapia no tratamento da alopecia androgenética. Brazilian Journal of Surgery and Clinical Research, [s. I.], v. 24, n. 2, p. 166-175, 2018

NEDEL, Wagner Luis et al. Os diferentes delineamentos de pesquisa e suas particularidades na terapia intensiva. Revista Brasileira de Terapia Intensiva, [s. I.], v. 28, n. 3, p. 256-260, 2016.

EL TAIEB, Moustafa A. et al. Platelets rich plasma versus minoxidil $\mathbf{5 \%}$ in treatment of alopecia areata: a trichoscopic evaluation. Dermatologic Therapy , [s. I.], p. 1-6, 2016.

GAN, D. C. C. et al. Prevalence of Male and Female Pattern Hair Loss in Maryborough. J Investig Dermatol Symp Proc, [s. I.], v. 10, p. 184-189, 2005.

POZEBON, Dirce et al. Análise de cabelo: uma revisão dos procedimentos para a determinação de elementos traço e aplicações. Química Nova, [s. I.], v. 22, n. 6, p. 838846, 1999.

OLIVEIRA, Inês de. Calvície e Alopécia. 2017. Dissertação (Mestre em Ciências Farmacêuticas) - Universidade Lusófona de Humanidades e Tecnologia, [S. I.], 2017.

CAVALCANTI, C. P. Protocolos de tratamento da alopécia: uma revisão. 2015. Dissertação (Graduação em Farmácia) - Universidade Estadual da Paraíba, [S. I.], 2015.

GAMONAL, Shirley. Tricologia. Revista Médica Oficial do Hospital Universitário da Universidade Federal de Juiz de Fora, [s. I.], v. 25, n. 2, p. 118-137, 1999.

MITEVA, Mariya. Alopecia. [S. I.]: Elsevier Editora, 2019.

SOARES, Anita Maria Pequeno. CABELO IMPORTA: os significados do cabelo crespo/ cacheado para mulheres negras que passaram pela transição capilar. 2018. Dissertação (Pós-graduação em Sociologia) - Universidade Federal de Pernambuco, [S. I.], 2018.

OMS: Metade da população masculina pode sofrer com a perda do cabelo até os 50 anos. Diário do Litoral, [S. I.], p. 1, 12 dez. 2018. Disponível em: https://

www.diariodolitoral.com.br/brasil/oms-metade-da-populacao-masculina-pode-sofrercom-a- perda-do-cabelo/120538/. Acesso em: 11 maio 2020.

REBELO, Ana Santos. Novas estratégias para o tratamento da alopécia. 2015. Dissertação (Mestrado em Ciências Farmacêuticas) - Universidade Lusófona de Humanidades e Tecnologia, [S. I.], 2015 
INJEÇÕES de PRP mecânico e controlada em pacientes afetados por alopecia androgenética. [S. I.: $\quad$ s. $\quad$ n.], 2018. Disponível em: https://www.jove.com/video/56406/injees-de-prp-mecnico- e-controlada-empacientes-afetados-por-alopecia?language=Portuguese. Acesso em: 15 maio 2020.

DISTRIBUIÇÃO da população por sexo, segundo os grupos de idade Distrito Federal. 2015. 1 Gráfico. Disponível em: https://censo2010.ibge.gov.br/sinopse/webservice/ frm_piramide.php?codigo $=53 \&$ corhomem $=3 d 4590 \&$ cormulher $=9 \mathrm{cdbfc}$. Acesso em: 15 maio 2020.

GOMES, N. L. Trajetórias escolares, corpo negro e cabelo crespo: reprodução de estereótipos ou ressignificação cultural? Revista Brasileira de Educação, Rio de Janeiro, n. 21, p. 40-51, 2002.

VOGT, Annika et al. Biology of the Hair Follicle, In: PEYTAVI, Ulrike Blume et al. Hair Growth and Disorders. Berlin: Springer, 2008. Cap. 1, p. 1-23.

VENDRAMIN, F. S. Método de obtenção do gel de plasma rico em plaquetas autólogo. Revista Brasileira de Cirurgia Plástica, [s. I.], v. 24, n. 2, p. 212-218, 2009.

ALVES, R. Randomized Placebo-Controlled, Double-Blind, Half-Head Study to Assess the Efficacy of Platelet-Rich Plasma on the Treatment of Androgenetic Alopecia. Dermatologic Surgery, [s. I.], v. 42, n. 4, p. 491-497, 2016.

SENNET, Rachel et al. Alopécia Androgenética. In: ALOPÉCIA. [S. I.: s. n.], 2019. 
ANEXOS

\section{ANEXO 1 - ANAMNESE}

\section{PROJETO DE INICIAÇÃO CIENTÍFICA - FAP/DF}

\section{Identificação:}

\begin{tabular}{|l|l|}
\hline Nome Completo & \\
\hline Idade & \\
\hline Data de Nascimento & \\
\hline Sexo & \\
\hline Profissão & \\
\hline Escolaridade & \\
\hline Cor & \\
\hline CPF & \\
\hline Endereço & \\
\hline Telefone para contato & \\
\hline Email & \\
\hline Nome da mãe & \\
\hline Examinador & \\
\hline Data & \\
\hline Local & \\
\hline
\end{tabular}

\section{Datas das consultas:}

$\mathbf{1}^{\mathrm{a}}$

$3^{2}$

\section{Check-List}

\begin{tabular}{|l|l|l|l|}
\hline \multicolumn{1}{|c|}{ ETAPA } & $1^{\mathrm{a}}$ & $2^{\mathrm{a}}$ & $3^{\mathrm{a}}$ \\
\hline 1. Explicar o projeto. & & & \\
\hline 2. Assinatura do TCLE. & & & \\
\hline 3. Anamnese. & & & \\
\hline 4. Questionário de autoavaliação. & & & \\
\hline 5. Inspeção (Hamilton e Norwood) & & & \\
\hline 6. Fotografia (F, T, P, O) & & & \\
\hline 7. Dermatoscopia & & & \\
\hline 8. PRP & & & \\
\hline 9. Orientações finais & & & \\
\hline
\end{tabular}

\section{Anamnese:}

QP:

1. Com quantos anos iniciou a calvície? 
2. Você notou algum fator desencadeante?

3. Qual foi o padrão de acometimento (mais comum é o H\&N)?

4. Você já realizou algum tratamento prévio? Qual? Obteve resultado?

5. Alguém na sua família apresentou perda de cabelo? Qual o motivo?

6. Já teve alguma infecção no couro cabeludo?

7. Possui o diagnóstico de tricotilomania? Tem o costume de puxar o cabelo?

8. Possui alguma alteração no processo de cicatrização?

9. Possui alguma comorbidade (Que tenham história prévia de malignidades, distúrbios plaquetários ou hemorrágicos, aplasia da medula óssea, diabetes, HIV, infecção por hepatite B ou C ou imunocomprometidos, tenham propensão a quelóides e hiperandrogenismo)? 

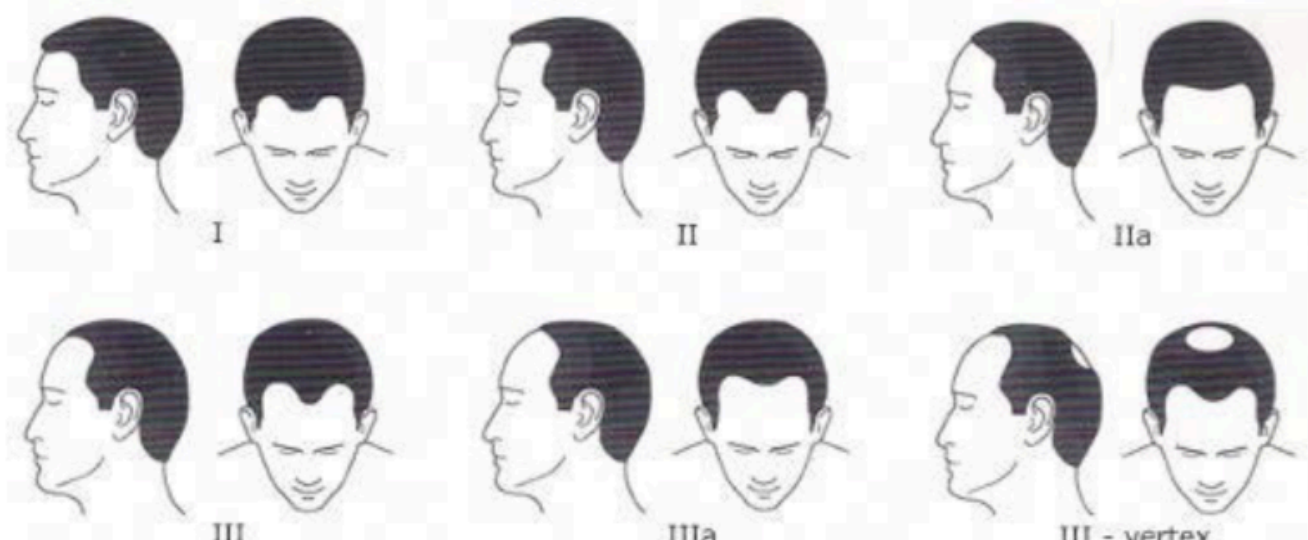

IIIa

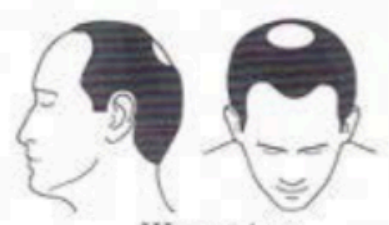

III - vertex
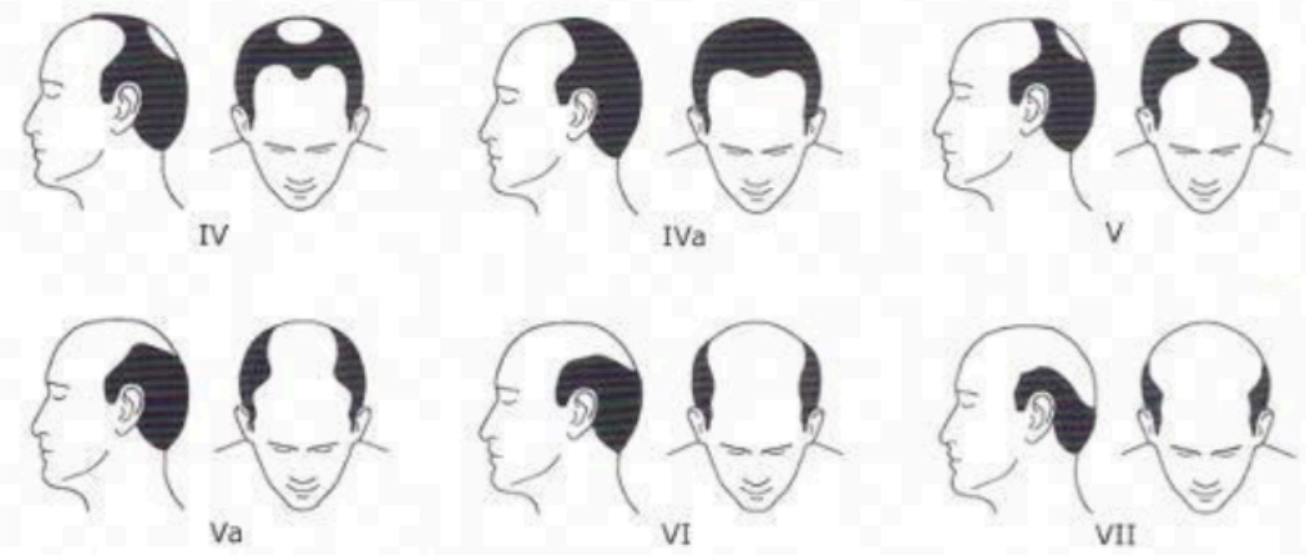


\section{ANEXO 3 - QUESTIONÁRIO DE AUTOAVALIAÇÃO}

\section{Questionário de autoavaliação}

\section{Preencher as perguntas de 1 a 6 apenas na $1^{\text {a }}$ Consultas}

1. Como você classificaria o nível de expectativa que tem com relação aos resultados deste estudo, de 0 a 5 , sendo " 0 " a mínima possível e " 5 " a máxima:

Responda as perguntas de 2 a 6 com: sempre, frequentemente, às vezes, raramente ou nunca.

2. Você se sente desconfortável, frustrado ou estressado por causa da Alopécia Androgenética (AAG)?

3. Você sente que a AAG piorou sua aparência física?

4. Você sente que que a AAG te afeta negativamente em sua faculdade ou local de trabalho?

5. Você tem deixado de comparecer a eventos sociais por causa da AAG?

6. Você sente que sua vida amorosa e sexual está sendo afetada pela AAG? 
Preencher as perguntas de 7 a 11 na segunda e terceira consulta:

7. Como você classificaria seu grau de satisfação com relação aos resultados obtidos até o momento, de 0 a 5 , sendo " 0 " a mínima possível e " 5 " a máxima:

8. Como você descreveria a sua percepção com relação à evolução do tratamento: abaixo do esperado, exatamente o esperado ou acima do esperado?

9. Você tem conseguido seguir as orientações da equipe rotineiramente? Se não, quais e por quê?

10. Você tem percebido sinais e sintomas que possam estar associados ao procedimento (exemplos seriam sinais e sintomas inflamatórios, como calor, vermelhidão, inchaço ou dor na região, ou qualquer outro que tem perdurado ou evoluído com a duração da pesquisa)? 
11. 0 tratamento gerou alguma mudança em seu cotidiano (na sua auto-estima, na opinião que você e os outros têm de você, entre outros fatores)? 